\title{
Criminologie
}

\section{La criminalité au Québec}

Daniel Élie

Volume 14, numéro 1, 1981

Criminalité et réalités sociales : Québec, Canada, États-Unis, Japon

URI : https://id.erudit.org/iderudit/017132ar

DOI : https://doi.org/10.7202/017132ar

Aller au sommaire du numéro

Éditeur(s)

Les Presses de l'Université de Montréal

ISSN

0316-0041 (imprimé)

1492-1367 (numérique)

Découvrir la revue

Citer cet article

Élie, D. (1981). La criminalité au Québec. Criminologie, 14(1), 85-104.

https://doi.org/10.7202/017132ar d'utilisation que vous pouvez consulter en ligne.

https://apropos.erudit.org/fr/usagers/politique-dutilisation/ 
Daniel Élie

Il est d'usage courant de mentionner l'état de la criminalité dans un territoire. Il existe des rapports officiels provenant de différentes sections d'une unité géographique et leur somme est censée foumir cette information. On est porté à traiter de l'état de la criminalité comme des comptes généraux de la nation, des mouvements de la Bourse ou du produit national brut. On ressent toujours, et on formule souvent, des réserves à l'égard de ces < comptes * mais le besoin d'informations quantitatives finit par convaincre et on \& utilise > les données soumises par les publications officielles.

C'est ainsi que le titre de cet article ne semble pas, en luimême et à première vue, soulever des questions bien exigeantes. En le considérant de plus près, toutefois, on peut se demander s'il est possible de dire un fait tel que * la criminalité au Québec $*$. Il peut être éclairant de s'attacher à cette question au préalable. Elle est examinée dans la première partie de ce texte.

En se demandant si la connaissance de l'état de la criminalité au Québec est possible, on interroge les moyens pouvant amener à cette connaissance, bien sûr, mais également la notion elle-même ou l'objet de connaissance. Il serait sans doute opportun d'établir une position par rapport à ce deuxième aspect de la question. En effet, la notion de criminalité semble avoir perdu un peu de sa transparence aux yeux de plusieurs. Ceci est dû, en partie du moins, aux développements mêmes de la criminologie. On peut référer spécifiquement sur ce point à la branche dite * radicale * de la discipline. Il n'est guère possible de s'attarder à ces considérations, fort intéressantes au demeurant, dans le court espace qui nous est alloue. Il faut cependant rapporter un des principaux thèmes de ce courant : la construction et 1'utilisation des indicateurs sociaux ne sont pas totalement indépendantes des choix politiques. Les statistiques sur la criminalité peuventelles être assimilées à des indicateurs sociaux? Si oui, ce qui précède signifie en termes clairs qu'elles ne parleraient pas de la criminalité de tous. Elles seraient influencées par des forces poli- 
tiques, par des valeurs et stéréotypes. Il s'agit d'une simple évidence qu'il fallait rappeler.

Nous mentionnons également que, dans ce texte, il faut entendre par statistiques sur la criminalité celles fournies par les différents corps de police. Le choix s'explique par le fait qu'elles constituent les données les plus stables et les plus importantes sur le sujet. Il n'existe pas au Québec, à notre connaissance, des sources d'informations complémentaires et d'égale valeur, telles les enquêtes dites de * victimisation *.

Afin de répondre à la question posée au sujet de nos possibilités de savoir, on doit maintenant examiner plus directement ces déclarations des administrations policières en tant que moyen de connaissance de la criminalité. Pour les comprendre, on est amené à considérer l'ensemble des facteurs qui interviennent dans leur production. En d'autres termes, il faut savoir comment un incident de nature criminelle peut faire partie de cette comptabilité. Ce résultat est le produit de nombreuses influences et nous en mentionnons quelques-unes en indiquant que ces chiffres dépendent :

a) de l'attitude des citoyens face à la criminalité, c'est-à-dire de leur intérêt et de leur assentiment à la signaler aux instances concernées ou présumées telles;

b) de la capacité de la police à découvrir des crimes ;

c) de ses manières d'y réagir et de les rapporter ;

d) de l'évolution des lois;

e) du type de société et des définitions légales utilisées;

f) des caractéristiques fondamentales de la population (ex. : la densité et l'importance démographique d'une collectivité);

g) plus récemment enfin, de la création de services de police et de sécurité privés.

Il faut par ailleurs retenir que l'influence et la qualité de ces facteurs ne sont pas nécessairement constantes. Par exemple, l'attitude des citoyens face à la criminalité peut varier, les habilités de la police également. On se trouve donc en présence d'un discours très difficile à comprendre et à interpréter. Dans l'état actuel des choses, on peut encore retenir l'idée, déjà vieille, que les statistiques officielles témoignent moins de la criminalité que des valeurs, intérêts et attitudes de ceux qui les compilent. 
En fait, les débats et interrogations autour des statistiques sur la criminalité ont commencé dès leur instauration en Europe depuis 1850. Déjà Adolphe Quételet (voir : Sellin, T. et Wolfgang, M. (1964)) déclarait :

... nos observations peuvent seulement référer à un certain nombre de crimes connus... du nombre total des crimes commis et inconnus. Comme cette somme totale demeurera probablement toujours inconnue, toute conclusion basée sur elle sera plus ou moins erronée.

Par la suite, il postule l'existence d'une relation quasi invariable entre les crimes connus et le total des crimes perpetrés. Ce postulat conférerait une valeur plus sûre aux statistiques sur la criminalité. Par contre, il admet également que cette relation varie selon la nature des crimes et selon les conditions sociales. Ce dernier point, la fluctuation des conditions sociales, est important parce qu'il oblige à interroger le postulat. En fait, celui-ci ne peut pas être raisonnablement accordé. La nature de la relation entre les crimes connus et le total des crimes réellement commis est difficilement décelable et rien ne permet d'affirmer l'existence d'un rapport invariable entre les deux sommes.

En 1929, le a Committee on Uniform Crime Records of the International Association of Chiefs of Police > admettait que les informations au sujet de certaines catégories de crimes (tel l'homicide) peuvent servir d'indice de l'évolution de la criminalité. Il reconnaissait implicitement que l'ensemble des données officielles ne remplissait pas nécessairement cette fonction.

Au Canada, Oosthoek (1978), après beaucoup d'autres, et même le Bureau fédéral de la statistique émettent des réserves au sujet des informations officielles sur la criminalité. On doute que ces données mesurent vraiment le phénomène qu'elles devraient mesurer.

Il est bien évident que mille répétitions ne font pas une vérité. Mais, en l'occurrence, il s'agit bien d'une vérité répétée depuis plus d'un siècle. Notre position peut être résumée comme suit.

Il est toujours imprudent de parler de a la Criminalité » parce que le terme désigne des faits trop différents, allant de la consommation occasionnelle de marijuana au meurtre prémé- 
dité. Nous questionnons donc la valeur et le sens des additions qu'il nous obligerait d'opérer. Lorsqu'on tient, malgré tout, à percevoir une telle chose, on constate qu'il n'est guère possible de décrire réellement la criminalité au Québec à partir des informations fournies par les administrations policières parce qu'on ne sait pas exactement ce qu'elles mesurent. catégories :

Ces données, par ailleurs, peuvent être divisées en deux

a) Des informations fiables qui constitueraient de véritables statistiques au sujet de certains crimes tels que : l'homicide, les vols de banque, les attentats à la bombe, les vols de véhicules automobiles; de manière générale, les incidents très « visibles 》 ou très fidèlement signalés, enregistrés et rapportés pour des raisons diverses (ex. : dédommagement par une compagnie d'assurance). La question concemant la valeur de ces crimes en tant qu'indices généraux (et de quoi ?) demeure ouverte ou nous la laissons telle.

b) Une autre série de données réunissant tous les autres crimes et infractions aux codes. Cette seconde catégorie peut être vue comme étant composée d'indicateurs de réaction sociale à un ensemble d'incidents et selon les groupes socio-économiques impliqués.

Ainsi, il nous semble plus raisonnable de considérer des études et informations portant sur certains types de crimes (ceux de la première catégorie) de manière spécifique en termes de réalités géographiques et de périodicité. Parmi les crimes pouvant avoir une valeur d'indice, nous avons retenu et présentons :

I. L'homicide à Montréal de 1944 à 1975. Il s'agit donc d'un fait à haut taux de signalement, observé durant une période relativement longue.

II. Les vols à main armée et les attaques contre les institutions bancaires. Ce type de crime semble caractériser la province de Québec et particulièrement le territoire relevant de la Communauté urbaine de Montréal.

\section{L'HOMICIDE $\lambda$ MONTREAL}

Au sujet de l'homicide à Montréal, les statistiques officielles sont relativement fiables, principalement à cause de la nature 
même de l'événement. Malgré l'existence d'un e chiffre noir *, elles traduisent l'évolution du phénomène en longue période. L'information la plus sûre demeure le nombre de victimes enregistrées.

Les statistiques que nous utilisons proviennent d'un relevé spécial fait par le Bureau de recherche et de planification de la Police de la Communauté urbaine de Montréal. Pour la période 1944 à 1975, on y trouve les informations suivantes : le nom de la victime, la date et le lieu de constat, le mode d'exécution, l'état du dossier, c'est-à-dire cause résolue ou non résolue par mise en accusation. Ces informations de base ont été vérifiées et complétées. Elles se limitent aux incidents survenus à l'intérieur des frontières de la ville de Montréal jusqu'en 1972 et celles de la Communauté urbaine de Montréal depuis sa fondation.

\section{L'importance relative de l'homicide}

La présence de l'homicide dans un milieu mobilise constamment une forte charge émotive. Dans l'opinion courante, à tort ou a raison, l'homicide tient la place du meilleur indicateur dans une économie de la violence du milieu. Ce statut d'indicateur provoque une perception privilégiée par rapport aux autres formes de violence et aux autres types de décès violents. Ainsi donc, dans un premier temps, il peut être éclairant de ne pas le considérer isolément mais de lui adjoindre des catégories de faits, de le situer parmi des ensembles. Il ne s'agit pas de banaliser le phénomène mais de faire un premier examen de son importance relative en termes de quantité. Comme catégories d'événements permettant d'atteindre ce but, nous utilisons les autres genres de décès violents et les autres crimes violents.

\section{L'importance de lhomicide par rapport aux autres types de} décès violents

Lo tableau I, ci-après, permet d'évaluer l'importance de l'homicide par rapport à cinq autres genres de morts violentes. La comparaison est faite durant une période de trois ans, soit de 1971,1973 et 1974. 


\section{TABLEAU I}

Meurtres et autres types de décès violents dans le district judiciaire de Montréal en 1971,1973 et 1974

(verdicts de morts accidentelles)

\begin{tabular}{lcccr}
\hline Types de décès & 1971 & 1973 & 1974 & Total \\
\hline Noyade & 59 & 70 & 89 & 218 \\
Asphyxie & 39 & 87 & 113 & 239 \\
Accident de travail & 33 & 32 & 83 & 148 \\
$\begin{array}{l}\text { Accident de véhicule } \\
\text { automobile }\end{array}$ & 344 & 355 & 310 & 1009 \\
Suicide & 187 & 153 & 191 & 531 \\
Meurtre & 59 & 52 & 78 & 189 \\
\hline Total & & & & 2334 \\
\hline
\end{tabular}

Sources : Annuaire du Québec 1973, p. 309-310.

Annuaire du Québec 1974, p. 407-408.

Annuaire du Québec 1975-76, p. 502-503.

Trois fois plus de gens meurent dans des accidents de la route que du fait d'un concitoyen. Si on recherche une cause de mort violente sensiblement aussi fréquente que l'homicide, on peut retenir les noyades et les accidents de travail industriel. Ils representent respectivement $9,3 \%, 6,3 \%$ du total. Les accidents de la route retiennent $43,2 \%$ des décès violents; les suicides officiellement enregistrés $22,75 \%$. Les homicides au cours de cette période constituent $8,09 \%$ des morts violentes sur l'île de Montréal.

L'importance de l'homicide par rapport d̀ la criminalité de violence

Le point est illustré au tableau II, ci-contre. On peut compléter la comparaison en retenant qu'au cours de cette période, la moyenne annuelle des infractions d'ordre sexuel, viol inclus, se situe à 1200 . Rappelons qu'il s'agit de crimes connus de la police, autrement dit, dans ce contexte, du nombre minimum. 


\section{TABLEAU II}

Crimes violents connus de la police durant la période 1972-1976. dans l'ile de Montréal

\begin{tabular}{lrrrrr}
\hline & 1972 & 1973 & 1974 & 1975 & 1976 \\
\hline Homicides & 89 & 45 & 68 & 101 & 84 \\
Tentatives de meurtre & 88 & 103 & 117 & 139 & 171 \\
Blessures & 48 & 88 & 137 & 86 & 52 \\
Voies de faits & 5457 & 5684 & 5833 & 6022 & 6006 \\
Vols qualifiés & 2783 & 3441 & 5165 & 7563 & 6742 \\
\hline Total : & 8465 & 9361 & 11320 & 13911 & 13055 \\
\hline
\end{tabular}

Il est bien évident que les faits retenus dans ces deux tableaux ne sont pas tous qualitativement comparables. On comprend que leurs nombres n'expriment pas leurs importances véritables dans un milieu, en ce sens qu'iis ne signalent pas les mêmes ordres de réalité ; ils n'amènent pas à la même lecture du milieu. Il s'agit simplement d'établir une évidence. L'homicide, en première analyse, se révèle comme étant un fait relativement rare à Montréal. Par rapport aux totaux du tableau II, il représente $0,5 \%$ des crimes violents en $1973,0,6 \%$ en 1974 et $0,7 \%$ en 1975.

La comparaison avec d'autres villes nord-américaines étaye ce point. Cette comparaison s'opère habituellement en référant au taux pour 100000 habitants d'une localité. Afin de contrôler, partiellement, l'effet des variations annuelles, on utilise parfois le taux annuel moyen de périodes caractéristiques. A Montréal pour 1974-1975, ce nombre est de 4,20 et ces deux années se situent au sommet de la série que nous présentons plus loin. On peut rapporter ce taux à ceux d'un certain nombre de villes américaines, pour la période 1971-1972 qui représente également selon les publications officielles, une phase d'accroissement du phénomène dans ce pays. On constate alors que le nombre de 4,20 est proche du taux signalé dans des villes telles que San José 
$(4,83)$, San Diego $(4,88)$, El Paso $(4,19)$. Par contre, durant cette période, le taux d'homicide dans tous les grands centres urbains des Etats-Unis est au minimum le quadruple de celui de Montréal. Ainsi, on rapporte 17,16 pour 100000 habitants à Boston, 21,74 à Philadelphie, 22,78 à Chicago, 34,39 à Washington, 38,93 à Détroit, 48,79 à Atlanta. Il existe donc une différence.

\section{L'évolution de l'homicide à Montréal}

La description du développement interne du phénomène consiste d'abord et essentiellement, dans ce contexte, à trouver une réponse à trois questions centrales, à savoir : Combien ? Quand ? et Où ? Les réponses n'étant pas satisfaisantes lorsqu'elles concernent une courte période de temps, nous utiliserons toutes les données disponibles. Elles couvrent une période de 31 ans.

Nous apportons des réponses en deux termes:

a) en premier lieu, Combien ? et Quand?, référant donc à l'évolution temporelle; et

b) en second lieu, Combien ?, Quand? et Ou ?, référant a l'évolution spatio-temporelle.

\section{a) L'évolution temporelle}

Cette information est rapportée au tableau III.

Avant de commenter ces données, il peut être utile d'éclairer la brusque augmentation du nombre de victimes en 1972. Elle s'explique très peu par la fondation de la Communauté urbaine de Montréal, c'est-à-dire par l'agrandissement du territoire et de

la population observée. On se rappellera de préférence qu'au cours de cette année, l'incendie criminel du Café Blue Bird a fait 37 victimes. La progression normale du phénomène, s'il est permis de s'exprimer ainsi, se rétablit au cours des années suivantes.

Il est bien clair que cette série présente trois périodes qu'on peut délimiter comme suit, en considérant le rythme d'accroissement du nombre de victimes : la première est comprise entre 1944 et 1955, la seconde entre 1956 et 1967 et la dernière entre 1968 
TABLEAU III

Nombre d'homicides enregistrés dans la ville de Montréal et dans la Communauté urbaine de Montréal de 1944 d 1975

\begin{tabular}{|c|c|c|}
\hline Années & Nombres d'homicides & $\begin{array}{c}\text { Taux par } 100000 \\
\text { habitants }\end{array}$ \\
\hline 1944 & 5 & 0,53 \\
\hline 1945 & 5 & 0,52 \\
\hline 1946 & 9 & 0,93 \\
\hline 1947 & 13 & 1,33 \\
\hline 1948 & 7 & 0,70 \\
\hline 1949 & 8 & 0,80 \\
\hline 1950 & 10 & 0,99 \\
\hline 1951 & 8 & 0,78 \\
\hline 1952 & 8 & 0,77 \\
\hline 1953 & 7 & 0,66 \\
\hline 1954 & 8 & 0,75 \\
\hline 1955 & 7 & 0,64 \\
\hline 1956 & 12 & 1,08 \\
\hline 1957 & 17 & 1,50 \\
\hline 1958 & 10 & 0,87 \\
\hline 1959 & 24 & 2,06 \\
\hline 1960 & 20 & 1,68 \\
\hline 1961 & 12 & 1,00 \\
\hline 1962 & 19 & 1,58 \\
\hline 1963 & 20 & 1,66 \\
\hline 1964 & 23 & 1,90 \\
\hline 1965 & 24 & 1,97 \\
\hline 1966 & 17 & 1,39 \\
\hline 1967 & 24 & 1,96 \\
\hline 1968 & 34 & 2,81 \\
\hline 1969 & 36 & 2,89 \\
\hline 1970 & 33 & 2,72 \\
\hline 1971 & 46 & 3,78 \\
\hline CUM 1972 & 89 & 4,51 \\
\hline 1973 & 45 & 2,28 \\
\hline 1974 & 68 & 3,39 \\
\hline 1975 & 101 & 5,02 \\
\hline Total & 769 & \\
\hline
\end{tabular}


et 1975. Celle-ci semble éloquente. En effet, une augmentation importante est nettement perceptible à partir de 1968. Les fluctuations de la densité de population pourraient être évoquées comme facteurs de correction à introduire dans l'interprétation des faits. Or, selon les données démographiques disponibles, la population de la ville de Montréal est demeurée relativement stable, avec une légère tendance à la baisse, de 1962 à 1972. Cette augmentation est donc antérieure à la fondation de la Communauté urbaine de Montréal et elle est observée dans une population stable. Aussi le tableau III n'illustrerait pas un e artefact > quelconque mais une augmentation réelle et importante du nombre de victimes.

Les fluctuations saisonnières de la criminalité ont parfois alimenté le folklore criminologique. Dans le jargon académique consacré, on parle de la loi thermique. Elle indique la prédominance des crimes contre les personnes durant les saisons chaudes et des crimes contre la propriété durant les saisons froides. Si on écarte les opinions trop subjectives et personnelles au sujet de la notion et du nombre réel de saisons à Montréal et si on y admet l'existence de quatre saisons bien distinctes, on dénombre $27,22 \%$ des homicides en hiver, $23,55 \%$ au printemps, $23,55 \%$ en été et $25,66 \%$ en automne. Il s'agit de variations fort modestes et peu concluantes. On s'en doutait bien, il n'existe pas une saison des meurtres.

\section{b) L'évolution spatio-temporelle}

Cette information est fournie au tableau IV. Nous retenons deux aspects de l'évolution spatio-temporelle du phénomène.

En premier lieu, il semble que la période 1961-1965 constitue une époque charnière, une sorte de tournant. On constate depuis une diffusion rapide du phénomène sur tout le territoire à partir du secteur centre de la ville. Ce fait est également remarquable au tableau IV qui présente la répartition des homicides dans la ville de Montréal par période de cinq ans, de 1944 à 1975 et selon les districts municipaux.

Quelques notes au sujet de ce tableau. Pour alléger la présentation, les noms des districts sont remplacés par des numéros. Pour la période de 1971-1975, il faut retenir que dans le district 
$\mathrm{n}^{\circ} 9$ (Centre) s'est produit l'incendie criminel du Café Blue Bird (37 victimes), dans le district $\mathrm{n}^{\circ} 24$, le massacre du Salon Bar Gargantua (13 victimes). Le tableau présente le nombre de victimes et non le nombre d'incidents. Au sujet du district $n^{\circ} 9$, désignant le quartier du Centre-Ville, il faut apporter quelques précisions. Ce district regroupe 6 des 38 quartiers municipaux définis par le Service des travaux publics de la ville de Montréal. Dans un Bulletin Technique publié en 1974, le Service d'urbanisme de la ville propose un nouvel encadrement du Centre-Ville qui est retenu au tableau IV. Il va de la rue Amherst à l'est aux limites de la cité de Westmount à l'ouest, de l'avenue des Pins au nord au fleuve Saint-Laurent au sud.

Il semble malaisé de dégager de ces informations un modèle très spécifique ou une image plus précise que celle d'une sorte d'explosion, d'une expansion presque aléatoire.

Le terme \& aléatoire > est relativement juste. En effet, on remarque, en second lieu, qu'il existe très peu de victimes d'homicides dans les zones à haut niveau socio-économique. Les secteurs tels que Westmount, Ville Mont-Royal, Outremont sont presque intacts, sauf à leurs périphéries. Le même fait a été constaté dans plusieurs villes nord-américaines. Il ne s'agit donc pas d'une particularité montréalaise.

On observe par ailleurs un certain changement parmi les moyens utilisés contre les victimes. Ils étaient relativement variés jusqu'au début de la période d'accroissement. Par contre, de 1963 à 1975 , un peu plus de la moitié des homicides $(52 \%)$ sont commis au moyen d'une arme à feu.

En 1976, on dénombre 85 victimes d'homicide, 101 en 1977, 73 en 1978. La série présente depuis sept ans une instabilité assez marquée. Une analyse des aspects du phénomène depuis 1975 n'entraîne aucune modification notoire à l'ensemble des faits exposés. Les mêmes constats reviennent, à savoir : un impact social plus diffus, plus généralisé, dont témoigne la répartition géographique; une certaine imperméabilité des zones sociales à haut niveau socio-economique et relativement homogènes; le caractère aléatoire de la diffusion dans les autres zones; la même hétérogénéité des mobiles apparents et une propension croissante à l'utilisation des armes à feu contre les victimes. 
TABLEAU IV

Distribution des homicides a Montréal par période de 5 ans, selon les districts, 1944-1975

\begin{tabular}{|c|c|c|c|c|c|c|c|}
\hline Districts * & $\begin{array}{l}1944^{* *} \\
1950\end{array}$ & $\begin{array}{l}1951 \\
1955\end{array}$ & $\begin{array}{l}1956 \\
1960\end{array}$ & $\begin{array}{l}1961 \\
1965\end{array}$ & $\begin{array}{l}1966 \\
1970\end{array}$ & $\begin{array}{l}1971 \\
1975\end{array}$ & Total \\
\hline 1 & 2 & 0 & 1 & 1 & 8 & 10 & 22 \\
\hline 2 & 0 & 2 & 3 & 4 & 6 & 8 & 23 \\
\hline 3 & 2 & 0 & 0 & 2 & 3 & 10 & 17 \\
\hline 4 & 0 & 0 & 0 & 0 & 4 & 4 & 8 \\
\hline 5 & 2 & 1 & 0 & 1 & 5 & 5 & 14 \\
\hline 6 & 1 & 0 & 1 & 2 & 2 & 2 & 8 \\
\hline 7 & 0 & 1 & 2 & 2 & 1 & 0 & 6 \\
\hline 8 & 0 & 1 & 3 & 4 & 1 & 7 & 16 \\
\hline 9 & 21 & 11 & 29 & 36 & 34 & 98 & 229 \\
\hline 10 & 1 & 1 & 4 & 2 & 4 & 3 & 15 \\
\hline 11 & 1 & o & 0 & 2 & 2 & 6 & 11 \\
\hline 12 & 0 & 0 & 3 & 4 & 4 & 5 & 16 \\
\hline 13 & 0 & 0 & o & 0 & 2 & 9 & 11 \\
\hline 14 & 0 & 1 & 3 & 5 & 1 & 17 & 27 \\
\hline 15 & 2 & 1 & 6 & 4 & 3 & 11 & 27 \\
\hline 16 & 1 & 1 & 3 & 4 & 2 & 1 & 12 \\
\hline 17 & 1 & 1 & 1 & 2 & 3 & 3 & 11 \\
\hline 18 & 2 & o & 1 & 2 & 1 & 4 & 10 \\
\hline 19 & 0 & 1 & 3 & 1 & 5 & 5 & 15 \\
\hline 20 & 2 & 1 & 0 & 1 & 2 & 5 & 11 \\
\hline 21 & 3 & 1 & 0 & 3 & 1 & 5 & 13 \\
\hline 22 & 1 & 0 & 1 & 1 & 5 & 6 & 14 \\
\hline 23 & 0 & 0 & 2 & 2 & 2 & 3 & 9 \\
\hline 24 & 0 & 0 & 2 & 1 & 6 & 25 & 34 \\
\hline 25 & 2 & 3 & 5 & 3 & 5 & 12 & 30 \\
\hline 26 & $\mathbf{0}$ & 1 & 0 & 1 & 2 & 2 & 6 \\
\hline 27 & 2 & 3 & 1 & 1 & 7 & 14 & 28 \\
\hline 28 & 0 & 0 & 1 & 1 & 2 & 7 & 11 \\
\hline 29 & 0 & 2 & 1 & 1 & 17 & 7 & 28 \\
\hline 30 & 0 & 0 & 0 & 0 & 0 & 0 & 0 \\
\hline 31 & 0 & 0 & 0 & 0 & 0 & 0 & 0 \\
\hline 32 & 0 & 0 & 0 & 0 & 1 & 4 & 5 \\
\hline
\end{tabular}

* On trouvera la liste des districts dans le \& Bulletin Technique de la Ville de Montréal * (1974).

** Selon les relevés disponibles pour cette période, il s'agit de six ans et non de cinq ans. 


\section{LES VOLs À MAIN ARMÉ ET LES ATtAQUES CONTRE LES INSTITUTIONS BANCAIRES}

On dispose à ce sujet de données fiables provenant de plusieurs sources, notamment la Sûreté du Québec, Statistique Canada, le Service de Police de la Communauté urbaine de Montréal, l'Association des Banques du Canada. Les nombres soumis ci-après reflètent convenablement l'état du phénomène au Québec et ailleurs dans le pays. Ils sont tirés du rapport du \& Groupe de travail sur les vols à main armée au Québec s, Ministère de la Justice, 1980.

Il est impossible de procéder à une description détaillée dans ces quelques pages, nous soumettons donc les points saillants parmi les informations disponibles.

Le tableau V ci-dessous permet de comparer la province de Québec et la Communauté urbaine de Montréal à l'ensemble du Canada.

\section{TABLEAU $\mathrm{V}$}

Vols qualifiés et vols à main armée

Evolution comparative : Canada, Québec, CUM (1974-1979)

\begin{tabular}{lcccccc}
\hline & \multicolumn{3}{c}{ Vols qualifés 1 } & \multicolumn{3}{c}{ Vols à main armée 2 } \\
& Canada & Québec & CUM & Canada & Québec & CUM \\
\hline 1974 & 16955 & 7953 & 5173 & 8630 & 5047 & 3312 \\
1975 & 21299 & 11167 & 7559 & 12455 & 7974 & 5531 \\
1976 & 20050 & 10356 & 6742 & 11261 & 7336 & 5040 \\
1977 & 19491 & 9790 & 6211 & 10854 & 6569 & 4512 \\
1978 & 19673 & 9620 & 6065 & 10533 & 6514 & 4358 \\
1979 & N.D. & 11050 & 7066 & N.D. & 7230 & 4833 \\
\hline
\end{tabular}

Sources : Statistique Canada, C.P.Q., S.Q., SPCUM.

1. Vols qualifiés : comprend le vol à main armée (art. 302d) et les autres formes de vols réalisés, avec ou sans armes, en utilisant la violence ou des menaces de violence (art. 302a, b et c du C.C.).

2. *Vols à main armée ": se définit comme le vol qualifié réalisé alors que l'agresseur \& est muni d'une arme offensive ou d'une imitation d'une telle arme , (art. 302d du C.C.). 
On constate ainsi qu'en 1975, par exemple, $52,4 \%$ des vols qualifiés et $64 \%$ des vols à main armée connus des polices canadiennes ont eu lieu au Québec. Par ailleurs, toujours par rapport au total pour l'ensemble du Canada, on notera que $35 \%$ des vols qualifiés et $44,4 \%$ des vols à main armée sont perpétrés sur le territoire de la Communauté urbaine de Montréal.

Il s'agit d'un fait massif qu'on ne peut expliquer, à notre connaissance, par le jeu des définitions légales ou des pratiques d'enregistrement des délits par les corps policiers. Pour le moment, on est porté à le considérer comme un aspect particulier de la criminalité au Québec.

Cette singularité est nettement soulignée par le tableau VI. On y relève que, durant l'année 1978, 6514 des 10533 vols à main armée ont été commis au Québec. La province d'Ontario, occupant le deuxième rang, suit bien loin en déclarant 2024 incidents.

La catégorie evols à main armée s dissimule, en fait, le nombre particulièrement élevé d'attaques contre les banques au Québec. C'est ce qui ressort très nettement de l'analyse du tableau VII. Il faut également y remarquer la tendance à l'accroissement du phénomène.

On pourrait penser que, sur ce point, la Communauté urbaine de Montréal est remarquable dans le contexte canadien mais banale par rapport aux grandes villes des Etats-Unis d'Amérique. Cette opinion doit être écartée après lecture du tableau VIII. On y constate qu'il se produit plus de vols de banques, en chiffre absolu, sur le territoire de la CUM que dans la ville de New York. On notera également le faible taux de solutions.

Il faut se rappeler que le nombre d'attaques contre les banques augmente régulièrement depuis 1962 dans plusieurs agglomérations urbaines d'Amérique du Nord (cf. Lunden, W.A., 1973). Ainsi, le Québec (et la CUM) participe à un mouvement d'ensemble. Or, vis-à-vis la croissance du nombre d'incidents on a pu observer les réactions de deux groupes concernés, celles de la police et celles des administrations bancaires.

La réaction des corps policiers du Québec en vue d'enrayer le nombre d'attaques a été assez violente. En effet, de 1968 à 1977, la police a été impliquée dans 26 fusillades ayant 
TABLEAU VI

Vols qualifies et vols a main armée par province Nombre et taux par 100000 habitants

\begin{tabular}{|c|c|c|c|c|c|c|c|c|c|c|c|c|}
\hline & \multicolumn{6}{|c|}{ Vols qualifies } & \multicolumn{6}{|c|}{ Vols a main armée } \\
\hline & 1974 & 1975 & 1976 & 1977 & 1978 & 1979 & 1974 & 1975 & 1976 & 1977 & 1978 & 1979 \\
\hline Terre-Neuve & $\begin{array}{l}59 \\
11\end{array}$ & $\begin{array}{r}41 \\
8\end{array}$ & $\begin{array}{r}41 \\
7\end{array}$ & $\begin{array}{r}50 \\
9\end{array}$ & $\begin{array}{l}53 \\
10\end{array}$ & & $\begin{array}{r}20 \\
4\end{array}$ & $\begin{array}{r}14 \\
3\end{array}$ & $\begin{array}{r}13 \\
2\end{array}$ & $\begin{array}{r}19 \\
3\end{array}$ & $\begin{array}{r}28 \\
5\end{array}$ & \\
\hline Ile-du-Prince-Edouard & $\begin{array}{l}13 \\
11\end{array}$ & $\begin{array}{r}10 \\
9\end{array}$ & $\begin{array}{l}9 \\
8\end{array}$ & $\begin{array}{l}14 \\
12\end{array}$ & $\begin{array}{l}8 \\
7\end{array}$ & & $\begin{array}{l}7 \\
6\end{array}$ & $\begin{array}{l}4 \\
4\end{array}$ & $\begin{array}{l}4 \\
3\end{array}$ & $\begin{array}{l}7 \\
6\end{array}$ & $\begin{array}{l}3 \\
3\end{array}$ & \\
\hline Nouvello-Ecosse & $\begin{array}{r}317 \\
39\end{array}$ & $\begin{array}{r}337 \\
43\end{array}$ & $\begin{array}{r}349 \\
42\end{array}$ & $\begin{array}{r}291 \\
35\end{array}$ & $\begin{array}{r}364 \\
44\end{array}$ & & $\begin{array}{r}116 \\
15\end{array}$ & $\begin{array}{r}120 \\
15\end{array}$ & $\begin{array}{r}136 \\
16\end{array}$ & $\begin{array}{r}107 \\
13\end{array}$ & $\begin{array}{r}128 \\
15\end{array}$ & \\
\hline Nouveau-Brunswick & $\begin{array}{l}97 \\
15\end{array}$ & $\begin{array}{l}99 \\
16\end{array}$ & $\begin{array}{r}132 \\
20\end{array}$ & $\begin{array}{r}116 \\
17\end{array}$ & $\begin{array}{r}140 \\
21\end{array}$ & & $\begin{array}{r}36 \\
6\end{array}$ & $\begin{array}{r}48 \\
8\end{array}$ & $\begin{array}{r}40 \\
6\end{array}$ & $\begin{array}{r}58 \\
9\end{array}$ & $\begin{array}{r}50 \\
7\end{array}$ & \\
\hline Québec & $\begin{array}{r}7953 \\
130\end{array}$ & $\begin{array}{r}11163 \\
180\end{array}$ & $\begin{array}{r}10356 \\
166\end{array}$ & $\begin{array}{r}9790 \\
157\end{array}$ & $\begin{array}{r}9620 \\
154\end{array}$ & $\begin{array}{r}11050 \\
177\end{array}$ & $\begin{array}{r}5047 \\
82\end{array}$ & $\begin{array}{r}7974 \\
128\end{array}$ & $\begin{array}{r}7336 \\
119\end{array}$ & $\begin{array}{r}6569 \\
105\end{array}$ & $\begin{array}{r}6514 \\
104\end{array}$ & $\begin{array}{r}7230 \\
116\end{array}$ \\
\hline Ontario & $\begin{array}{r}3840 \\
47\end{array}$ & $\begin{array}{r}4350 \\
53\end{array}$ & $\begin{array}{r}4323 \\
52\end{array}$ & $\begin{array}{r}4447 \\
54\end{array}$ & $\begin{array}{r}4690 \\
57\end{array}$ & & $\begin{array}{r}1528 \\
20\end{array}$ & $\begin{array}{r}1968 \\
26\end{array}$ & $\begin{array}{r}1836 \\
22\end{array}$ & $\begin{array}{r}2088 \\
27\end{array}$ & $\begin{array}{r}2024 \\
26\end{array}$ & \\
\hline Manitoba & $\begin{array}{r}635 \\
63\end{array}$ & $\begin{array}{r}691 \\
70\end{array}$ & $\begin{array}{r}635 \\
62\end{array}$ & $\begin{array}{r}669 \\
66\end{array}$ & $\begin{array}{r}669 \\
66\end{array}$ & & $\begin{array}{r}211 \\
21\end{array}$ & $\begin{array}{r}231 \\
23\end{array}$ & $\begin{array}{r}230 \\
23\end{array}$ & $\begin{array}{r}193 \\
19\end{array}$ & $\begin{array}{r}267 \\
26\end{array}$ & \\
\hline Saskatchewan & $\begin{array}{r}307 \\
34\end{array}$ & $\begin{array}{r}330 \\
36\end{array}$ & $\begin{array}{r}345 \\
38\end{array}$ & $\begin{array}{r}349 \\
38\end{array}$ & $\begin{array}{r}356 \\
39\end{array}$ & & $\begin{array}{r}80 \\
9\end{array}$ & $\begin{array}{l}92 \\
10\end{array}$ & $\begin{array}{r}104 \\
11\end{array}$ & $\begin{array}{r}109 \\
12\end{array}$ & $\begin{array}{r}106 \\
12\end{array}$ & \\
\hline Alberta & $\begin{array}{r}1155 \\
67\end{array}$ & $\begin{array}{r}1450 \\
89\end{array}$ & $\begin{array}{r}1454 \\
79\end{array}$ & $\begin{array}{r}1646 \\
90\end{array}$ & $\begin{array}{r}1358 \\
74\end{array}$ & & $\begin{array}{r}516 \\
32\end{array}$ & $\begin{array}{r}651 \\
40\end{array}$ & $\begin{array}{r}635 \\
35\end{array}$ & $\begin{array}{r}822 \\
45\end{array}$ & $\begin{array}{r}503 \\
27\end{array}$ & \\
\hline Colombie-Britannique & $\begin{array}{r}2538 \\
106\end{array}$ & $\begin{array}{r}2789 \\
128\end{array}$ & $\begin{array}{r}2328 \\
94\end{array}$ & $\begin{array}{r}2093 \\
85\end{array}$ & $\begin{array}{r}2387 \\
97\end{array}$ & & $\begin{array}{r}1069 \\
49\end{array}$ & $\begin{array}{r}1353 \\
62\end{array}$ & $\begin{array}{r}927 \\
36\end{array}$ & $\begin{array}{r}888 \\
36\end{array}$ & $\begin{array}{r}910 \\
37\end{array}$ & \\
\hline Territoires du Nord-Ouest & 41 & 39 & $\begin{array}{r}78 \\
*\end{array}$ & 26 & 28 & & & & & & & \\
\hline Canada & $\begin{array}{r}16955 \\
76\end{array}$ & $\begin{array}{c}21299 \\
93\end{array}$ & $\begin{array}{r}20050 \\
87\end{array}$ & $\begin{array}{r}19491 \\
84\end{array}$ & $\begin{array}{r}19673 \\
84\end{array}$ & & $\begin{array}{r}8630 \\
39\end{array}$ & $\begin{array}{r}12455 \\
55\end{array}$ & $\begin{array}{r}11261 \\
49\end{array}$ & $\begin{array}{r}10854 \\
47\end{array}$ & $\begin{array}{r}10533 \\
45\end{array}$ & \\
\hline
\end{tabular}

- Estimation par différence. Source : Commission de Police du Québec. 


\section{TABLEAU VII}

Vols d̀ main armée Banques faisant partie de l'ABC $1970-1979$

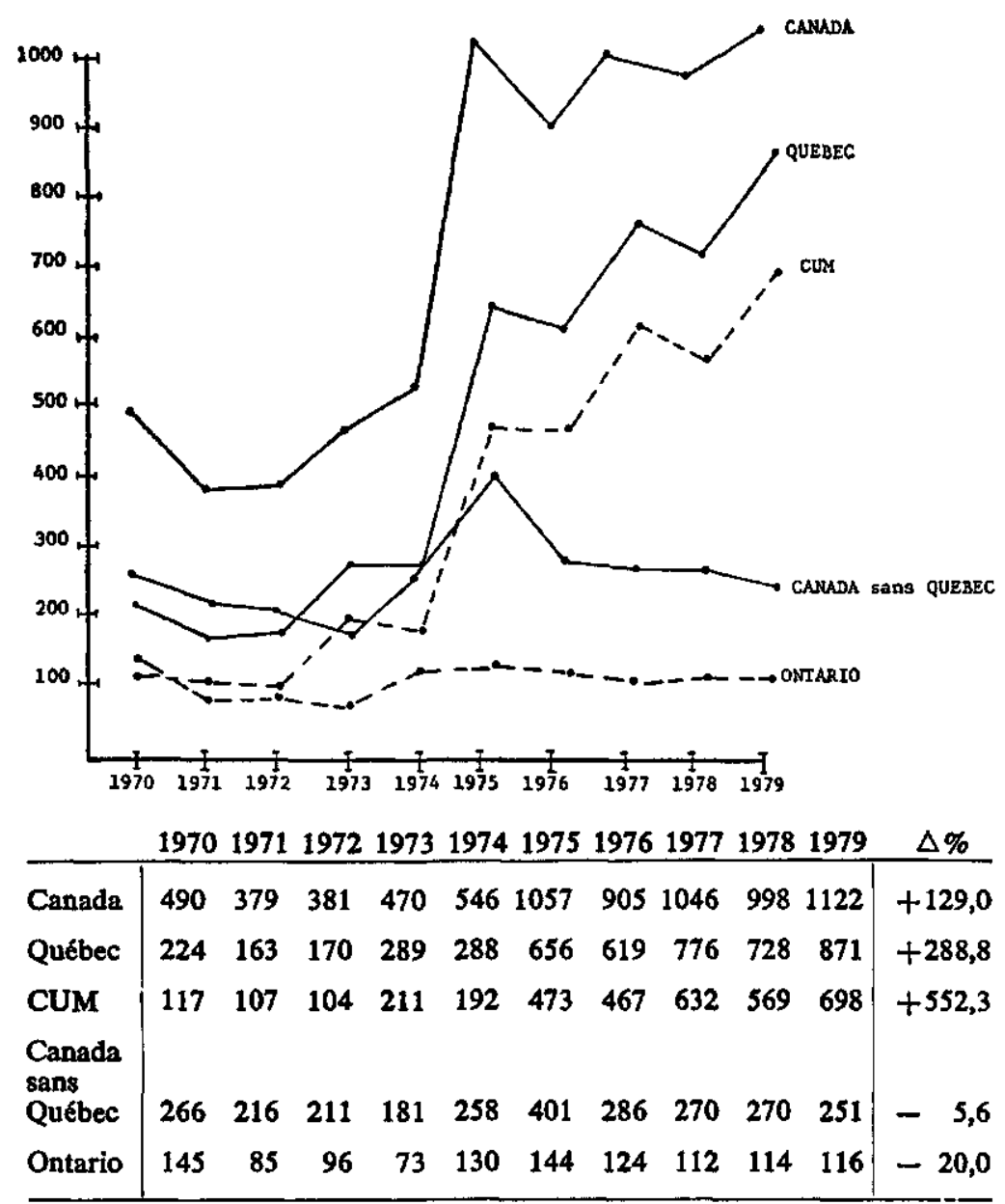

$\begin{array}{lllllllllll}\text { \% Québec 47,5 } & 43,0 & 44,6 & 61,5 & 52,7 & 62,1 & 68,4 & 74,2 & 72,3 & 77,6\end{array}$

Source : L'Association des banquiers canadiens.

entraîné le décès de 31 bandits ou présumés tels, soit le nombre le plus élevé au Canada. On constate que 11 de ces fusillades ont eu lieu à l'occasion de vols à main armée dans une banque ou 
TABLEAU VIII

$V$ ols de banque de certaines villes nord-américaines 1976

\begin{tabular}{|c|c|c|c|c|c|c|c|}
\hline & \multirow[b]{2}{*}{ Population } & \multirow[b]{2}{*}{$\begin{array}{c}\text { Total } \\
\text { vol qualifié }\end{array}$} & \multicolumn{2}{|c|}{ Vol de banque } & \multicolumn{3}{|c|}{ Vol de banque classé } \\
\hline & & & Nombre & $\begin{array}{c}\text { Taux par } \\
100000 \\
\text { habitants }\end{array}$ & Nombre & $\%$ & $\begin{array}{l}\% \text { VDB par } \\
\text { rapport au } \\
\text { total de VQ }\end{array}$ \\
\hline Boston & 618000 & 6125 & 29 & 4,7 & $?$ & $?$ & 0,5 \\
\hline Chicago & 3365000 & 17577 & 35 & 1,0 & 23 & 66 & 0,2 \\
\hline Détroit & 1510000 & 21213 & 48 & 3,2 & $?$ & $?$ & 0,3 \\
\hline Los Angeles & 2825000 & 14314 & 319 & 11,3 & 195 & 61 & 2,2 \\
\hline New York (1975) & 7900000 & 83190 & 443 & 5,6 & 179 & 40 & 0,5 \\
\hline San Francisco & 668000 & 6640 & 119 & 17,8 & 37 & 31 & 1,8 \\
\hline St. Louis & 535000 & 5303 & 14 & 2,6 & 14 & 100 & 0,3 \\
\hline Toronto & 2190000 & 1774 & 75 & 3,4 & 39 & 52 & 4,2 \\
\hline Washington & 730000 & $\begin{array}{l}8134 \\
(1975)\end{array}$ & 82 & 11,2 & 60 & 73 & 1,0 \\
\hline CUM & 1835000 & 6742 & 589 & 32,1 & 181 & 31 & 8,7 \\
\hline
\end{tabular}

Source : PCUM 
une caisse populaire. De plus, diverses autres mesures (systèmes d'alarme, caméra, patrouilles, etc.) ont rendu ce type de délit plus dangereux pour l'agresseur.

Les banques, par ailleurs, ont réagi en réduisant notamment les sommes d'argent manipulées aux guichets. On estime actuellement qu'il faut perpétrer environ cinq attaques contre une banque pour se procurer un montant de 10000 dollars. Il s'agit donc d'une activité criminelle qui est devenue non seulement plus risquée mais aussi peu payante; une entreprise de petits truands et d'amateurs.

Les bandits recherchant des profits substantiels en une seule opération ont réagi à leur tour en kidnappant des gérants de banques ou leurs proches. De 1974 à 1978, on mentionne dans la région de Montréal, une quarantaine de ces incidents, mais la pratique des prises d'otages et enlèvements pour fin de rançon a cessé depuis deux ans.

Les faits exposés ci-dessus réfèrent à une période relativement brève, mais la situation décrite n'est pas nouvelle. Il s'agit d'une caractéristique stable. En effet, en 1970, la Commission Prévost signalait déjà le nombre remarquablement élevé de vols à main armée et d'attaques contre les banques au Québec et soumettait une quarantaine de recommandations pour contrôler ce type de crimes. L'ensemble des mesures adoptées ont été inopérantes de manière très évidente et rien actuellement ne laisse présager une résorbtion du phénomène.

\section{CONCLUSION}

Reconsidérons en conclusion le rôle de ces deux types de crimes comme indicateurs de la criminalité générale. On constate une certaine correspondance entre l'aspect global de l'évolution de l'homicide et celui de la somme des délits connus, telle que décrite par les statistiques officielles. Mais le fait n'ayant pas été suffisamment observé, il peut être fortuit et ne saurait constituer l'argument le plus valable.

Le tableau est différent en ce qui concerne les vols à main armée. La fréquence, la permanence et l'accroissement du phénomène distingue nettement la province de Québec par rapport à l'ensemble du Canada. Par ailleurs, son évolution et celle de la 
criminalité générale déclarée ne sont pas isomorphes. Donc, dans l'état actuel de nos connaissances, la question de leur valeur en tant qu'indices demeure ouverte.

Enfin, lorsqu'on examine l'ensemble des problèmes soulevés au sujet de la connaissance exacte du volume de la criminalité, on est tenté de se poser la question suivante : est-il vraiment nécessaire d'atteindre une précision infinitésimale sur ce point? Tout probablement non. Par contre, il serait sans doute raisonnable d'accorder an moins une égale importance à la perception de la criminalité par les citoyens ainsi qu'aux modifications apportées par cette perception à leur façon d'être et de vivre.

\section{BIBLIOGRAPHIE}

BOUDOWRIS, James (1974) : A Classification of Homicides ?, Criminology, 11, 525-40.

BROWN, Robert (1963) : Explanation in Social Science, Chicago, Aldin Publishing Co.

BULLOCK, Henry A. (1955) : - Urban Homicide in Theory and Fact s, Journal of Criminal Law, Criminology and Police Science, 45, 565-75.

ERICKSON, M.L. et GIBBS, J.P. (1979) : Community Tolerance and Measures of Delinquency ", Journal of Research in Crime and Delinquency, 16.1, 55-80.

ERICKSON, M.L.; GIBBS, J.P. et JENSEN, G. (1977) : - Conventional and Special Crime and Delinquency Rates \#, Journal of Criminal Law and Criminology, 68, 440-453.

KITSUSE, John I. et CICOUREI, Aaron J. (1963) : - A Note on the Use of Official Statistics ", Social Problems, 11, 131-39.

LOFLAND, Lyn H. (1973) : A World of Strangers : Order and Action in Urban Public Space, New York, Basic Books, Inc.

LUNDEN, W.A. : : The Bank Robbery Boom ", Security World, January 1973.

MACDONALD, John M. (1961) : The Murderer and his Victim, Springfield (IIl.), Charles C. Thomas Publ.

NOBLE, J.V. (1977) : A Feedback, Instability and Crime Waves s, Journal of Research in Crime and Delinquency, 16.1, 107-128. 
OOSTHOEK, A. (1978) : Utilisation des statistiques sur la criminalité, Solliciteur général du Canada, Division de la recherche.

RAPPORT DE LA COMMISSION D'ENQUETE PRÉVOST (1970).

SELLIN, T. et WOLFGANG, M.E. (1964) : The Measurement of Delinquency, New York, John Wiley and Sons Inc.

SELLIN, T. (1951) : e The Significance of Records of Crime *, The Law Quarterly Review, 67, 489-504.

TAFT, Donald R. (1966) : * Influence of the General Culture on Crime *, Federal Probation, 30, 16-23.

TOCH, Hans (1969) : Violent Men : An Inquiry into the Psychology of Violence, Chicago, Aldin Publishing Co. 